\title{
Fatigue of Metallic Stents: From Clinical Evidence to Computational Analysis
}

\author{
F. Auricchio, ${ }^{1}$ A. Constantinescu, ${ }^{2}$ M. Conti, ${ }^{1}$ and G. Scalet $^{1,2}$ \\ ${ }^{1}$ Dipartimento di Ingegneria Civile e Architettura, Università di Pavia, Via Ferrata 3, 27100 Pavia, Italy; and ${ }^{2}$ Laboratoire de \\ Mécanique des Solides - CNRS UMR 7649, École Polytechnique, 91128 Palaiseau, France
}

\begin{abstract}
The great success of stents in treating cardiovascular disease is actually undermined by their long-term fatigue failure. The high variability of stent failure incidence suggests that it is due to several correlated aspects, such as loading conditions, material properties, component design, surgical procedure, and patient functional anatomy. Numerical and experimental non-clinical assessments are included in the recommendations and requirements of several regulatory bodies and they are thus exploited in the analysis of stent fatigue performance. Optimization-based simulation methodologies have been developed as well, to improve the fatigue endurance of novel designs. This paper presents a review on the fatigue issue in metallic stents, starting from a description of clinical evidence about stent fracture up to the analysis of computational approaches available from the literature. The reported discussion on both the experimental and numerical framework aims at providing a general insight into stent lifetime prediction as well as at understanding the factors which affect stent fatigue performance for the design of novel components.
\end{abstract}

Keywords-Metallic stent, Stent fatigue, Clinical evidence, Computational analysis, Fatigue criteria.

\section{INTRODUCTION}

Stents are medical devices used to restore the lumen patency of stenotic arteries, i.e., arteries narrowed by atherosclerosis, a degenerative disease of the vessel wall. The stent is a tube-like lattice structure acting as a mechanical scaffold for the vessel. Its implantation is performed by a minimally invasive procedure exploiting endovascular catheters. ${ }^{31}$ Currently, $85 \%$ of coro-

Address correspondence to G. Scalet, Dipartimento di Ingegneria Civile e Architettura, Università di Pavia, Via Ferrata 3, 27100 Pavia, Italy. Electronic mail: giulia.scalet@unipv.it nary interventions involve stents, ${ }^{31}$ adding up to more than 1 million stents per year. ${ }^{53}$

The successful clinical use of stents has highlighted issues associated with long-term endurance of implants. ${ }^{3}$ Fractured stents lose their ability to scaffold occluded arteries and may cause restenosis, ${ }^{75}$ thrombosis, ${ }^{15}$ or artery perforation. ${ }^{52}$

Stent failure can be caused by the mechanical loading, either monotonic or cyclic loading during deployment or service, respectively. Cyclic loading is due to the pulsatile blood pressure or to vessel movements imposing bending, torsion, or tension/compression on the stent. Other factors can contribute to stent failure, such as material properties, stent design, physiological environment, surgical procedure, patient functional anatomy and lifestyle. ${ }^{67}$

In the following, we will refer to the failure due to cyclic loading as fatigue. Although the word fatigue is widely used in the engineering community, it is rarely adopted in the medical community. As a clear example, if you type the keyword 'stent fracture' in PubMed $^{1}$, you get 1001 papers; on the contrary, if you type 'stent fatigue', you get only 198 papers, most of them written from an engineering point of view.

Stents are small scale devices subjected to hundreds millions of in-service loading cycles and thus direct experimental testing is difficult to perform. As an example, accelerated pulsatile testing at $65 \mathrm{~Hz}$ takes about 72 days to complete $4 \times 10^{8}$ cycles. ${ }^{53}$ Therefore, computer-based design modeling represents an assessment tool for the prediction of stent performance and lifetime. ${ }^{25,47}$ Such a tool allows for the improvement of

\footnotetext{
${ }^{1}$ http://www.ncbi.nlm.nih.gov/pubmed.
} 
both clinical procedures and stent design and it is now also accepted by regulatory bodies. ${ }^{45,84}$

The present paper aims at providing an overview on the fatigue issue in metallic stents manufactured from stainless steel, cobalt chromium, and Nitinol-based shape memory alloys. The paper covers both the clinical evidence of stent fractures and the computational design approaches for lifetime assessment. We expect to bridge the gap between the engineering and medical communities. The goal of the work is to resume and to critically discuss the existing framework available from the literature and to open up new questions and future perspectives for the optimization of stent design, materials, and clinical procedures.

\section{CLINICAL EVIDENCE}

Stent failure and fracture (SF) are an inherent risk of stenting, having clinical consequences. ${ }^{1}$ The risk of endovascular SF is increased by unfavorable biomechanical actions depending on the stent positioning in vascular sites: stents in coronary arteries are subjected to mechanical stretching from repetitive heart contractions, while stents in femoral, popliteal, and carotid arteries undergo repetitive conformational deformations, i.e., traction and bending, imposed by body movement. ${ }^{1}$

We now briefly discuss recent clinical evidences on SFs among the arterial regions often treated by stent-


Femoro-popliteal

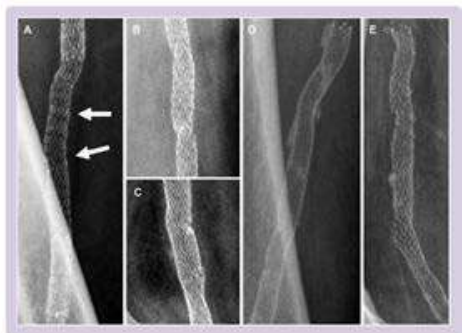

FIGURE 1. Illustrative representation of the main arterial districts treated by stenting. The stent size, design, and mechanical features depends on the specific artery to be treated. (left) Reprinted from Ref. 64, with permission from Elsevier; (top-right) reprinted from Ref. 22, with permission from Elsevier; (middle-right) reprinted from Ref. 27, with permission from John Wiley and Sons; (bottom-right) reprinted from Ref. 54, with permission from SAGE publications. 

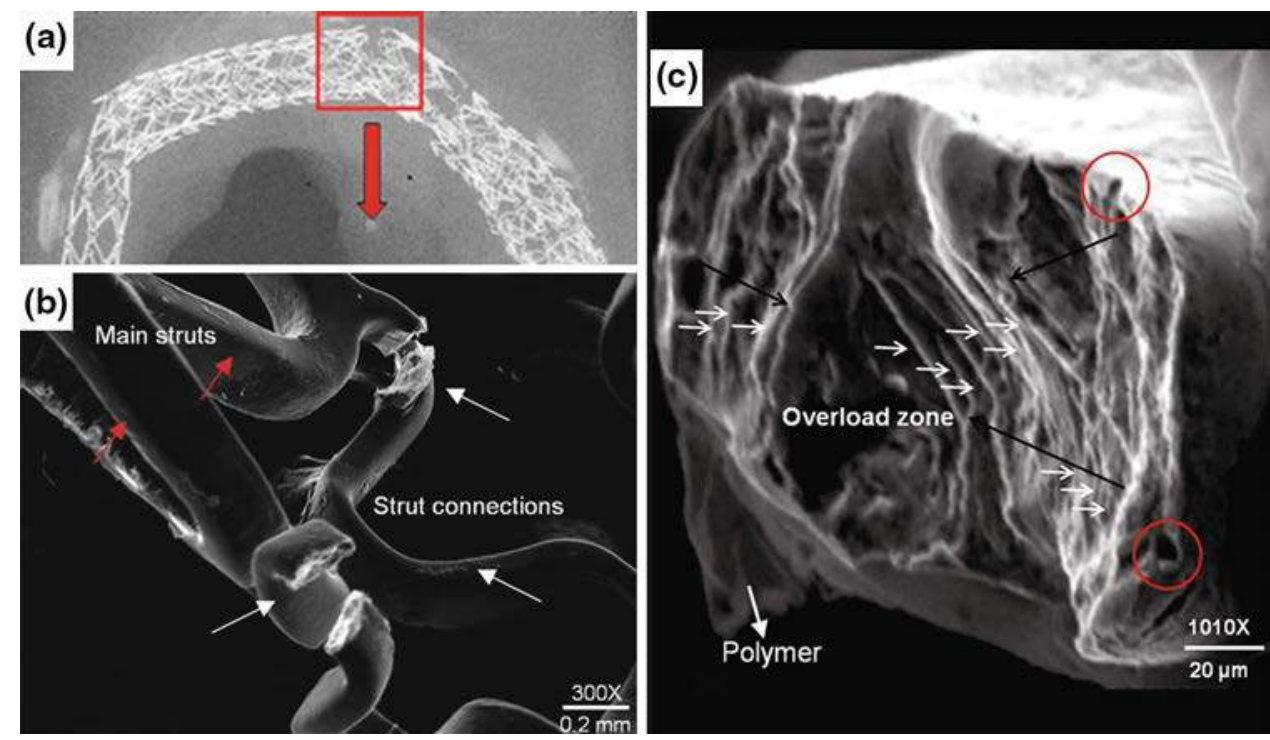

FIGURE 2. Multiple overlapping stents placed in the right coronary artery for 7 years. (a, b) All fractures occurred at the bend of connecting links. (c) Representative fracture demonstrating multiple high-cycle fatigue crack initiation and propagation sites surrounding the final overload zone, indicating the effect of bending forces. The irregular shape of the overload zone indicates the presence of multiple forces, possibly due to the stent overlap and/or calcification (red circles: possible fatigue crack initiation sites; small white arrows: high-cycle fatigue striations; black arrows: direction of fatigue crack propagation). Reprinted from Ref. 38, with permission from John Wiley and Sons.

ing (see Fig. 1), using the SF classification system recently proposed in Ref. 20.

\section{Coronary Arteries}

The cardiac contraction induces a consistent movement of coronary arteries toward the left, inferior, and anterior directions. ${ }^{76} \mathrm{SF}$ incidence varies between different studies; as an example, it is reported in $1-2 \%$ of patients at only 8 - to 10 -month follow-up angiography; higher rate (up to $29 \%$ ) after patient death (see Refs. 1,3,38 and references therein). It has been observed that long or multiple stents are subjected to higher radial forces and consequently to higher fracture risk (see Fig. 2), especially in the presence of calcification or deployment in angulated vessels (see Ref. 1 and references therein).

\section{Femoro-popliteal Arteries}

Given their position, femoro-popliteal vessels are subjected to additional external forces due to walking and leg bending, compression, torsion, and elongation. ${ }^{18}$ Data reported in Ref. 75 clearly indicate a relation between the vessel biomechanics and the fracture incidence of first generations of Nitinol femoral stents: $24.5 \%$ stents showed fractures, classified as minor (fracture of one strut) in $48.4 \%$ cases, moderate (fracture of several struts) in $26.6 \%$ cases, and severe (complete separation of stent segments) in $25 \%$ cases. Fracture rates were 13.2, 42.4, and $52.0 \%$ when correlated to stented lengths of $8,8-16$, and $16 \mathrm{~cm}$, respectively. Restenosis of $50 \%$ was found in $32.8 \%$ of the SF cases and a complete reocclusion was found in $34.4 \%$ of the SF cases. Although new generations of stents ${ }^{2}$ have increased the long-term durability of such implants, ${ }^{29}$ failure is still a matter of clinical debate. ${ }^{61}$

\section{Carotid, Renal, and Iliac Arteries}

Fractures of carotid stents are uncommon events, ${ }^{30}$ despite there are a free floating position of the artery subjected to torsion, flexion, and extension ${ }^{1}$ and an additional loading caused by swallowing and side-toside head turning. ${ }^{70}$ However, the rate of SF or deformation was 15 and $50 \%$ at 2 and 4 years and has been associated with the presence of heavy calcifications. ${ }^{16}$ Similarly, only a limited number of renal artery (see Ref. 71 and references therein) and iliac artery ${ }^{40}$ SFs has been reported.

\section{RECOMMENDATIONS AND REQUIREMENTS BY REGULATORY BODIES}

Regulatory bodies provide precise recommendations or requirements for the non-clinical validation of stents and associated delivery systems. ${ }^{45,84}$

Particularly, such indications consider mechanical stress/strain and fatigue analyses as well as accelerated durability testing for the evaluation of the in-vivo stent performance. Testing results are obtained imposing 10 
year equivalent pulsatile cycles on a mock artery with a deployed stent within a biological-equivalent environment (see, e.g., test systems ${ }^{44}$ ) and are completed by failure analysis, together with the definition of fatigue criteria and safety factors. Numerical and experimental analyses will be discussed in more detail in next sections.

\section{STENT MATERIALS AND DESIGNS}

In view of the regulations discussed in "Recommendations and Requirements by Regulatory Bodies" section, rigorous numerical and experimental testing is required for the development and verification of stents. The aspects to consider when developing a stent are its material and design, which are strictly connected and strongly affect the performance of the final device. Stent fatigue performance is mainly related to component dimensions and geometry, in-service loading, physiological conditions, material microstructure, surface condition, heat treatment, and manufacturing process. ${ }^{3}$ We now briefly discuss the effect of materials and design on fatigue performance.

\section{Stent Materials}

Common material choices are metals as $316 \mathrm{~L}$ stainless steels, L605 cobalt chromium, or Nitinolbased shape memory alloys, since they present essential features as suitable mechanical properties for deployment and service, excellent corrosion resistance, and biocompatibility. ${ }^{3,19}$ When compared in terms of fatigue resistance, L605 cobalt chromium alloys present a higher fatigue strength than stainless steel. Descriptions of fatigue and fracture mechanisms in superelastic Nitinol alloys remain incomplete even today, see, e.g., Ref. 72 for a review. For all these alloys both the cyclic hysteresis response and the fatigue behavior are sensitive to the microstructure and, particularly, to grain size. Fine-grained materials have been shown to be resistant to fatigue crack initiation, while coarsegrained materials are more resistant to fatigue crack growth. For stents, fatigue crack initiation dominates lifetime and, therefore, a fine-grained material is highly desirable (see Ref. 80 and references therein). Conventional thermomechanical processes can reduce the grain size up to approximately $10 \mu \mathrm{m}$ for $316 \mathrm{~L}$ stainless steel or cobalt chromium alloys and up to $100 \mathrm{~nm}$ for Nitinol alloys. As a consequence, the average number of grains per cross-section is 10 for stainless steel and cobalt chromium alloys, compared to 1000 for Nitinol alloys. $^{72}$
TABLE 1. Stent designs analyzed or tested in fatigue literature.

\begin{tabular}{lll}
\hline Material & \multicolumn{1}{c}{ Stent design } & \multicolumn{1}{c}{ Ref. } \\
\hline \multirow{2}{*}{ 316L stainless steel } & PalmazShatz & Refs. 26,33 \\
& Cypher & Refs. 26,48 \\
& Medinol NIR ${ }^{\text {TM }}$ & Refs. 5,79 \\
& JostentCFlex & Ref. 87 \\
Cobalt chromium alloys & Medinol NIR ${ }^{\text {TM }}$ & Ref. 82 \\
& Express & Ref. 48 \\
Nitinol alloys & Maris Plus & Ref. 68 \\
& IsoStent & Ref. 33 \\
& Cordis SMART Control & Refs. 11,63,66 \\
& Protégé EverFlex & Ref. 63 \\
& Luminexx & Ref. 63 \\
& LifeStent FlexStar & Ref. 63 \\
& Xceed & Ref. 63 \\
& Absolute & Ref. 63 \\
\hline
\end{tabular}

The table lists only the works explicitly reporting the investigated stent design

\section{Stent Designs}

Stents are periodic assemblies of repeated unit cells and are manufactured either through welding of microscopic wires or laser cutting from thin-walled tubes. Both the manufacturing processes lead to a final truss-type structure composed of struts connected by hinges, with small cross-sections and radii $(50-150 \mu \mathrm{m})$ and large inclusions $(\sim 15 \mu \mathrm{m})$, affecting stent performance due to high stress concentrations. ${ }^{3,88}$

Several stent designs, analyzed or tested in the fatigue literature, are reported in Table 1 . We remark that some designs are no longer in use (e.g., Cypher or PalmazShatz), despite the recent date of the studies. The works permit however a critical comparison of the evolution of the design methods and are an illustration of the growing market and of the need to continuously update the analysis.

\section{COMPUTATIONAL APPROACHES FOR THE LIFETIME ASSESSMENT OF STENTS}

Computational approaches are widely exploited in the design process of stents and are also considered by regulatory bodies. ${ }^{45,84}$

Computational methods for the lifetime assessment of stents are discussed based on a shakedown analysis of the fatigue phenomena, as treated in, e.g., Ref. 21. More precisely, the shakedown analysis classifies the mechanical stress-strain response of a structure under cyclic loading in three main categories: (i) elastic shakedown if the response is linear and no hysteretic loop is observed, (ii) plastic shakedown if the response exhibits a hysteretic loop, and (iii) ratcheting if the 




FIGURE 3. Schematic representation of the fatigue phenomenon at the macroscopic and mesoscopic scales of a generic stent in relation to the number of cycles to failure.

response is a non-closed path drifting to larger-andlarger strains. These classes are precise a signature of energy dissipation and of the eventual underlying damage mechanism, i.e., elastic shakedown is a reversible cycle with no energy dissipation, whereas the other two categories exhibit energy dissipation.

Failure and fatigue are associated to irreversible material processes and are thus characterized by energy dissipation. Energy dissipation can be considered as a measure of the damage which is initially created at the microstructure scale and subsequently develops in crack and flaws inducing the complete failure of the stent. Such a remark shows that the shakedown analysis should be applied both at the microscopic and the macroscopic scale of the structure. The schematic view of the described analysis and the different situations in terms of low- or high-cycle lifetime are displayed in Fig. 3. The reader is referred to section S1 of Supplementary Materials for details about the phenomenon of fatigue.

As a consequence of the reported considerations, the methodology adopted to assess stent lifetime can be denoted as a global computational approach and consists of two steps:

1. a mechanical analysis to calculate the stabilized state of the stent under cyclic loading and to assess the shakedown state. It can be performed using different computational techniques, such as structural finite element analysis, and it is based on given geometry (stent design, see "Stent Materials and Designs" section), loading conditions (see "Mechanical Analysis of Stents" section), and material constitutive law (see "Mechanical Analysis of Stents" section).

2. a fatigue analysis to compute the number of cycles to failure through appropriate fatigue criteria from the previously computed stabilized cycle (see "Fatigue Analysis of Stents" section).

The distinction between the mechanical and fatigue analyses assumes that the constitutive behavior is decoupled from the evolution of damage. Such an approximation permits to speed up computations and it has provided excellent prediction results in various engineering applications, e.g., Ref. 4. Recall, however, that alternative approaches based on the continuum damage mechanics theory have been also been applied to stents, e.g., Ref. 26.

The lifetime assessment considered in the following is essentially a prediction of crack initiation. Crack propagation will be neglected in our discussion as the 
number of cycles in this phase is generally far smaller than the complete lifetime of the stent. Moreover, the fatigue analysis will consider the failure of the stent when a single crack will initiate in a hinge or strut, since it will present a risk for the stenting procedure.

\section{MECHANICAL ANALYSIS OF STENTS}

The mechanical analysis should simulate as accurately as possible stent material properties, design, and the entire processing history, i.e., manufacturing, crimping on the delivery system, expansion/deployment, recoil, and physiological loading. We first focus on the physiological loading conditions and then on the material constitutive modeling.

\section{Physiological Loading Conditions}

The in-vivo loading conditions of stents and the corresponding number of cycles per year are summarized from ${ }^{41,50}$ in Table 2 . Table 3 reports the loading conditions adopted in stent simulations and Fig. 4

TABLE 2. Standard in-vivo loading conditions for stents, which depend on stent positioning in the human body (see "Clinical Evidence" section).

\begin{tabular}{llll}
\hline Loading & Frequency $(\mathrm{Hz})$ & Cycles per year & Ref. \\
\hline Diastole-systole & 1.2 & $4 \times 10^{7}$ & Ref. 41 \\
Musculoskeletal & 1 & $\sim 0.2 \times 10^{7}$ & Ref. 50 \\
Breathing & 0.3 & $\sim 1 \times 10^{7}$ & Ref. 41 \\
\hline
\end{tabular}

Recall that the 10-year equivalent cycles required by regulatory bodies (see "Recommendations and Requirements by Regulatory Bodies" section) translate in $4 \times 10^{8}, 2 \times 10^{7}$, and $1 \times 10^{8}$ cycles for diastole-systole, musculoskeletal, and breathing loading, respectively. presents four schematic examples of loading conditions. All the reported loading conditions are cyclic, i.e., assume a constant amplitude. The pulsatile pressure is generally assumed to range between 60 and 160 $\mathrm{mmHg}$. The values of compression and bending loading are usually defined according to clinical evidences, e.g., studies on cadavers. ${ }^{41,67}$ Such clinical data generally refer only to specific case-studies and we do not dispose of detailed patient-specific databases to assess these values. The imposed cyclic character of the loading can be extended to variable amplitude loading as it is has already been done on engineering structures, ${ }^{46}$ but no discussion in this sense has been found for stents.

Other important loading configurations impacting stent lifetime stem from the presence of atherosclerotic plaques, calcifications, or stent overlapping configurations. Although the risk of fracture is high in case of overlapping stents, ${ }^{75}$ due to fretting fatigue, there is only one work investigating this issue, i.e., Ref. 57 (see Table 3).

\section{Material Constitutive Modeling}

Material constitutive laws can be presented in the general form:

$$
\boldsymbol{\sigma}=\boldsymbol{\sigma}(\boldsymbol{\varepsilon}, \boldsymbol{\alpha}) \text { and } \dot{\alpha}=\dot{\alpha}(\boldsymbol{\varepsilon}, \boldsymbol{\alpha})
$$

where $\boldsymbol{\varepsilon}$ is the total strain, $\boldsymbol{\sigma}$ the stress tensor, $\boldsymbol{\alpha}$ the internal variable designating inelastic deformation, phase change, etc. and $\dot{\alpha}$ the time derivative of $\boldsymbol{\alpha}$.

The main constitutive laws used in stent computations stem from the class of standard continuum mechanics. Constitutive models can be classified as follows: (a)

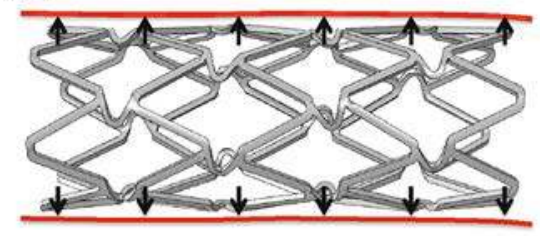

(c)

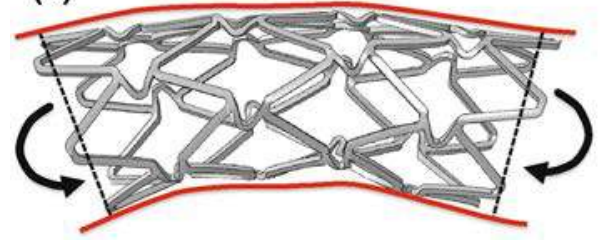

(b)



(d)

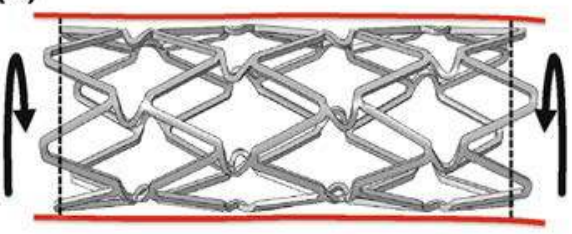

FIGURE 4. Schematic examples of cyclic loading conditions adopted in the mechanical analysis of stents. (a) Diastole-systole; (b) compression; (c) bending; and (d) torsion. 
TABLE 3. Adopted loading conditions in stent fatigue literature.

\begin{tabular}{|c|c|c|c|c|c|c|c|c|}
\hline Material & Cyclic loading & Fully-expanded & $\begin{array}{l}\text { Straight } \\
\text { vessel }\end{array}$ & $\begin{array}{l}\text { Curved } \\
\text { vessel }\end{array}$ & $\begin{array}{l}\text { Overlapping } \\
\text { stents }\end{array}$ & Plaque & $\begin{array}{c}\text { Plaque } \\
\text { calcification }\end{array}$ & Ref. \\
\hline \multirow{4}{*}{$\begin{array}{l}316 \mathrm{~L} \\
\text { stainless } \\
\text { steel }\end{array}$} & Diastole-systole & & & & & & & Ref. 56 \\
\hline & Diastole-systole & & $\mathrm{x}$ & & & & & Refs. 5'13,26,79 \\
\hline & Diastole-systole & & $x$ & & & $x$ & & Refs. 10,12 \\
\hline & Bending & $\mathrm{x}$ & & & & & & Ref. 5 \\
\hline \multirow{5}{*}{$\begin{array}{l}\text { Cobalt } \\
\text { chromium } \\
\text { alloys }\end{array}$} & Diastole-systole & & & & & & & Ref. 53 \\
\hline & Diastole-systole & & $x$ & & & & & Refs. 55,82 \\
\hline & Diastole-systole & & & $x$ & & $x$ & & Ref. 58 \\
\hline & Diastole-systole & & & $x$ & & & $x$ & Ref. 58 \\
\hline & Diastole-systole/bending & & & & & & & Refs. 41,42 \\
\hline Nitinol & Diastole-systole & & & & & & & Ref. 43 \\
\hline \multirow[t]{12}{*}{ alloys } & Diastole-systole & & $x$ & & & & & Ref. 39 \\
\hline & Diastole-systole & & & $x$ & & & & Ref. 39 \\
\hline & Diastole-systole & & $x$ & & & $x$ & & Refs. 57,67 \\
\hline & Bending & & $x$ & & & & & Ref. 39 \\
\hline & Bending & & $x$ & & & $x$ & & Ref. 39 \\
\hline & Bending & & & $x$ & & & & Ref. 39 \\
\hline & Torsion & & $x$ & & & & & Ref. 39 \\
\hline & Torsion & & & $x$ & & & & Ref. 39 \\
\hline & Compression & $x$ & & & & & & Ref. 57 \\
\hline & Compression & & $x$ & & & & & Ref. 57 \\
\hline & Compression & & $x$ & & & $x$ & & Refs. 24,67 \\
\hline & Compression & & $\mathrm{x}$ & & $\mathrm{x}$ & & & Ref. 57 \\
\hline
\end{tabular}

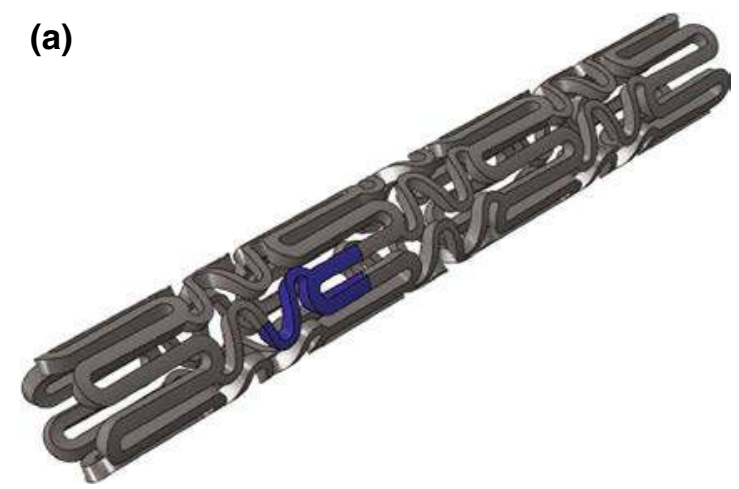

(b)

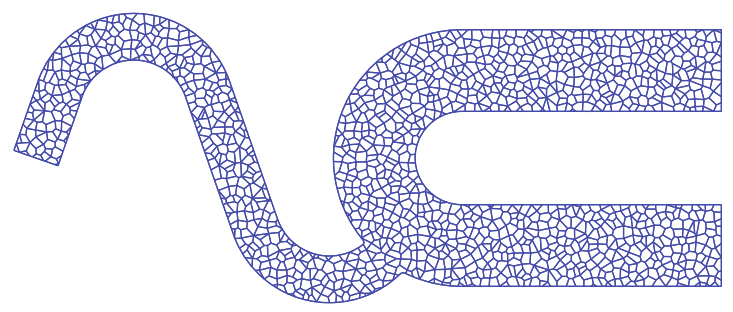

FIGURE 5. (a) Cypher stent geometry used in the three-dimensional homogeneous model (the blue domain highlights one of the unit cells). (b) Example of a unit cell geometry used in the two-dimensional micromechanical model, containing approximately 1500 grains (personal communication of Dr. Raphael Guerchais).

- Macroscopic phenomenological models, describing the macroscopic mechanical behavior and capturing microstructural effects only through phenomenological internal variables, as plastic or transformation strain.

- Crystal plasticity models, describing the mechanical behavior at the length scale of the grains. These models have an important computational expense as the mesh should include the precise grain distribution (see Figs. 5a and $5 b)$.

- Strain-gradient plasticity models, accounting for size-scale-dependent material behavior. Such models allow to take into account grain size effect on material hardening and fatigue behavior.

A summary of the models used in the stent literature is presented in Table 4. Recall that the choice of a constitutive model represents an important step of the mechanical analysis, especially when dealing with components where size effects are significant. Moreover, the adopted material model should simulate both monotonic and cyclic material behavior to account for the crimping, deployment, and cyclic loading. 
TABLE 4. Adopted constitutive models in stent fatigue literature.

\begin{tabular}{|c|c|c|}
\hline Material & Constitutive model & Ref. \\
\hline \multirow[t]{5}{*}{$316 \mathrm{~L}$ stainless steel } & Rate-independent $J_{2}$ perfect plasticity & Ref. 26 \\
\hline & Rate-independent $J_{2}$ plasticity with isotropic hardening & Refs. $10,12,13$ \\
\hline & Rate-independent $J_{2}$ plasticity with nonlinear kinematic/isotropic hardening & Refs. 5,79 \\
\hline & Rate-dependent crystal plasticity with Taylor isotropic hardening & Ref. 56 \\
\hline & $\begin{array}{l}\text { Rate-dependent crystal plasticity with nonlinear isotropic and Armstrong- } \\
\text { Frederick kinematic hardening }\end{array}$ & Ref. 79 \\
\hline \multirow[t]{4}{*}{ Cobalt chromium alloys } & Rate-independent $J_{2}$ plasticity with isotropic hardening & Refs. 53,58 \\
\hline & Rate-independent $J_{2}$ plasticity with nonlinear kinematic/isotropic hardening & Ref. 82 \\
\hline & $\begin{array}{l}\text { Rate-dependent crystal plasticity with nonlinear isotropic and Armstrong- } \\
\text { Frederick kinematic hardening }\end{array}$ & Refs. 81,82 \\
\hline & Rate-dependent strain-gradient crystal plasticity & Refs. 80,81 \\
\hline \multirow[t]{5}{*}{ Nitinol alloys } & Auricchio-Taylor-Lubliner ${ }^{9,69}$ & Refs. $24,39,43,57,66,67$ \\
\hline & Souza-Auricchio ${ }^{8,78}$ & Ref. 6 \\
\hline & Lagoudas et al. ${ }^{51}$ & Ref. 34 \\
\hline & Zaki-Moumni $^{91}$ & Ref. 59 \\
\hline & Single-crystal model ${ }^{28}$ & Ref. 86 \\
\hline
\end{tabular}

TABLE 5. Parameters for the dissipated energy criterion in stent fatigue literature.

\begin{tabular}{|c|c|c|c|c|}
\hline Material & Approach & $b$ & $c(\mathrm{MPa})$ & Ref. \\
\hline \multirow[t]{2}{*}{ 316L stainless steel } & Hot spot & -0.4687 & 431.4 & Ref. 7 \\
\hline & Volumetric (radius $0.01 \mathrm{~mm}$ ) & -0.6187 & 907.8 & Ref. 7 \\
\hline Nitinol alloys & Hot spot & -0.3770 & 11.0 & Ref. 59 \\
\hline
\end{tabular}

\section{FATIGUE ANALYSIS OF STENTS}

The fatigue analysis is based on criteria which permit to compute stent lifetime. Such criteria are based on a damage parameter which characterizes the stabilized cycle obtained from the mechanical analysis and which can be correlated with lifetime. The criteria are then calibrated using appropriate experiments (see "Fatigue Testing of Stents" section).

In the literature various uniaxial and multiaxial fatigue criteria have been applied to stents, as discussed in the following. Recall that all the discussed criteria predict crack initiation. Complementary approaches considering crack propagation are based on flaw-tolerant criteria which have been successfully applied to larger structures, e.g., orthopedic implants. Their application to stents ${ }^{55,73}$ can be exploited for quality control procedures during manufacturing to establish inspection pass/failure criteria for use during visual inspection of parts for surface defects. However, crack size inspection for implanted devices is not possible and the cost of implant failure is high.

\section{Uniaxial Fatigue Criteria}

This class includes criteria based only on stress or strain components associated with privileged directions of specific experiments. A criterion commonly adopted is the Goodman criterion, as also recommended in Ref. 84. It is defined in terms of stresses as:

$$
\frac{\sigma_{a}}{\sigma_{-1}}+\frac{\sigma_{m}}{\sigma_{u}} \leq 1
$$

where $\sigma_{a}, \sigma_{m}, \sigma_{u}$, and $\sigma_{-1}$ are the stress amplitude, mean stress, ultimate tensile stress, and fully reversed fatigue limit, respectively. Several works have proposed Goodman analyses for $316 \mathrm{~L}$ stainless steel, ${ }^{10,12}$ L605 cobalt chromium, ${ }^{41,42,53}$ and Nitinol ${ }^{24,43,57}$ stents. The three-dimensional application of this criterion is generally based on the use of the first principal strains/ stresses as index of risk. ${ }^{24}$ However, since the stent is subjected to a multiaxial state of solicitations, the criterion should consider equivalent mean and alternate stresses/strains. ${ }^{74}$ Moreover, the Goodman relationship is generally conservative for ductile metals and it is not directly applicable to Nitinol alloys. ${ }^{83}$

\section{Multiaxial Fatigue Criteria}

This class covers criteria based on invariant quantities extracted from the complete tensorial variables as stress, strains, or plastic strain.

A criterion commonly applied to the low-cycle fatigue regime is the dissipated energy criterion, defined as: ${ }^{4}$

$$
W^{p}=c N_{f}^{b}
$$


TABLE 6. Parameters for the Dang Van criterion in stent fatigue literature.

\begin{tabular}{|c|c|c|c|c|c|}
\hline Material & $N$ & Width $(\mu \mathrm{m})$ & $\alpha_{N}$ & $\beta_{N}(\mathrm{MPa})$ & Ref. \\
\hline \multirow[t]{5}{*}{$316 \mathrm{~L}$ stainless steel } & $2 \times 10^{6}$ & - & 1.50 & 420.0 & Ref. 13 \\
\hline & $4 \times 10^{8}$ & 50 & 0.4821 & 111.93 & Ref. 5 \\
\hline & $4 \times 10^{8}$ & 75 & 0.4821 & 137.50 & Ref. 5 \\
\hline & $4 \times 10^{8}$ & 100 & 0.4821 & 146.84 & Ref. 5 \\
\hline & $4 \times 10^{8}$ & 150 & 0.4821 & 165.0 & Ref. 5 \\
\hline Nitinol alloys & $1 \times 10^{7}$ & - & 0.808 & 137.77 & Ref. 6 \\
\hline
\end{tabular}

TABLE 7. Parameters for the microstructure-sensitive approach in stent fatigue literature.

\begin{tabular}{llllll}
\hline Material & \multicolumn{1}{c}{ Approach } & \multicolumn{1}{c}{ Constitutive model } & $p_{\text {crit }}(-)$ & $w_{\text {crit }}\left(\mathrm{MJ} / \mathrm{m}^{3}\right)$ & Ref. \\
\hline 316L stainless steel & Local (discrete) & Crystal plasticity & 43.3 & 12636 & Ref. 79 \\
Cobalt chromium alloys & Local (discrete) & Crystal plasticity & 439.9 & 156300 & Ref. 82 \\
& Non-local (averaged) & Crystal plasticity & 200.8 & 73200 & Ref. 81 \\
& Local (discrete) & Strain-gradient crystal plasticity & 164.3 & 91900 & Ref. 80 \\
& Non-local (averaged) & Strain-gradient crystal plasticity & 123.8 & 63900 & Ref. 81 \\
\hline
\end{tabular}

where the dissipated energy per cycle $W^{p}=\int_{\text {cycle }} \boldsymbol{\sigma}: \dot{\boldsymbol{\varepsilon}}^{p} \mathrm{~d} t$ is computed over the complete stabilized cycle, $\varepsilon^{p}$ being the plastic strain, and $b$ and $c$ are material parameters (see Table 5). The criterion has been successfully applied in Ref. 7 to match experimental results on notched specimens used in stent manufacturing. ${ }^{23,89}$

Another criterion successfully applied to the highcycle fatigue regime is the Dang Van criterion, ${ }^{85}$ which is based on a multiscale approach and shakedown concepts. The criterion states that lifetime is infinite if the mesoscopic shear stress, $\tau_{\mu}(t)$, and the hydrostatic stress, $\sigma_{\mathrm{h}}(t)$, satisfy the following inequality (subscript $\mu$ stands for mesoscopic variables):

$$
\max _{t}\left[\tau_{\mu}(t)+\alpha_{N} \sigma_{\mathrm{h}}(t)\right] \leq \beta_{N}
$$

$\alpha_{N}$ and $\beta_{N}$ being material parameters, where subscript $N$ stands for finite lifetime (see Table 6). The multiscale characteristic of the criterion allows to take into account the different phases of shape memory alloys, ${ }^{6}$ based on the shakedown theorems in Ref. 65 .

Recently, approaches based on microstructure-sensitive fatigue indicator parameters and used in conjunction with crystal plasticity theory have been introduced to take into account microstructural inhomogeneities, as follows:

$$
\left\{\begin{array}{l}
N_{i}=\frac{p_{\text {crit }}-p_{\text {recoil }}}{p_{\text {cyc }}} \\
N_{i}=\frac{w_{\text {crit }}-w_{\text {recoil }}}{w_{\text {cyc }}}
\end{array}\right.
$$

where $N_{i}$ is the number of cycles to crack initiation; $p_{\text {crit }}, p_{\text {recoil }}$, and $p_{\text {cyc }}$ are the accumulated plastic slip values at crack initiation (calibrated), at the beginning of deployment, and during one fatigue cycle, respectively; $w_{\text {crit }}, w_{\text {recoil }}$, and $w_{\text {cyc }}$ are the corresponding values for strain energy dissipation (see Table 7).

\section{Difficulties and Perspectives}

A first difficulty is that the inhomogeneous nature of the microstructure, which determines a significant scatter in fatigue, ${ }^{80}$ is neglected by most of the cited criteria, in spite of the small scale of the strut and the low number of grains. The Goodman criterion does not consider size effects nor high gradients. The Dang Van criterion successfully meets the need of microstructural representation in crack initiation predictions, but cannot capture inhomogeneity effects observed in random crystalline microstructure modeling. On the contrary, the techniques based on a microstructural analysis of the inhomogeneities, coupled with crystal plasticity and stress-gradient models, have successfully captured the fatigue scatter. ${ }^{79-81}$

Another difficulty is the presence of small curvature radii in stent struts, which are responsible for high stress concentrations in the presence of a heterogeneous microstructure. This has motivated the adoption of volumetric approaches (see Tables 5 and 7), the theory of critical distance, ${ }^{10}$ as well as stress-gradient crystal plasticity models. ${ }^{80,81}$

To overcome such difficulties, the presence of heterogeneous microstructures has been discussed in Ref. 14 for the Dang Van criterion and extensions including a probabilistic setting have been proposed in Ref. 37. The dissipated energy criterion has been combined with a defect growth law and a probabilistic analysis of the microstructure defects to account for material variability in Ref. 17. These approaches ad- 


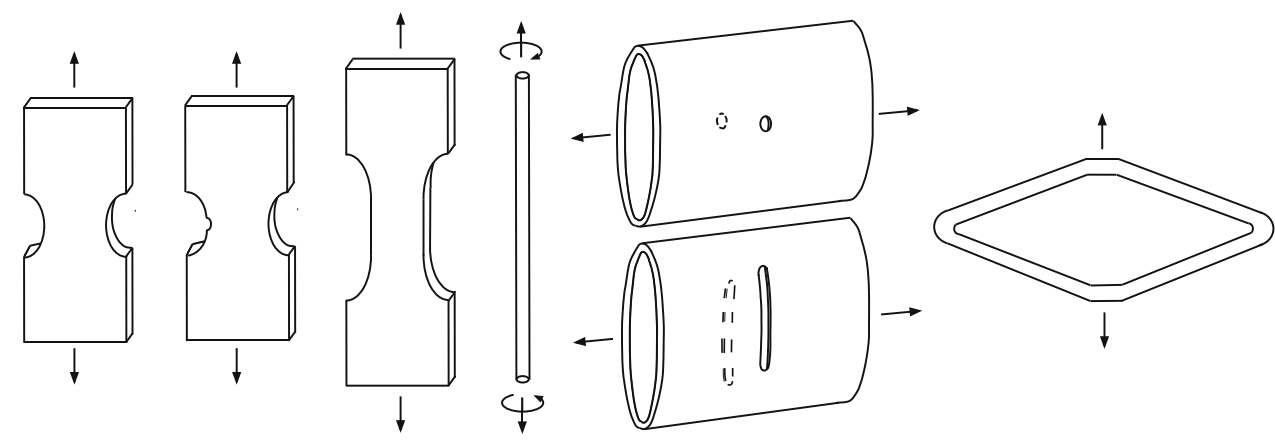

FIGURE 6. Geometries of the microscopic components usually tested in the literature and corresponding loading conditions; see, e.g., Refs. $66,74,82,88$.

dress the scatter issue related with microstructural inhomogeneities and could be future perspectives for stent lifetime prediction.

\section{FATIGUE TESTING OF STENTS}

Fatigue testing, combined with computational analysis, allows to provide an indication of stent durability. It can be used for the validation of the fatigue analysis and for the identification of the conditions that are not modeled using computational methods. ${ }^{45,84}$

Accelerated durability testing is usually conducted on stents deployed inside synthetic arteries in specially designed rigs simulating pulsatile loading, body temperature, and salinity; see, e.g., Refs. 33,48,49,60,63,87. The physiological environment can be phosphate-buffered saline, Ringer's, or Hank's solution at $37^{\circ} \mathrm{C}$. Loading frequencies range from 20 to $100 \mathrm{~Hz}$ (the normal heart rate is approximately $1.2 \mathrm{~Hz}$ ). Other types of cyclic loading (e.g., bending, compression) or different configurations of stents (e.g., overlapping) or synthetic arteries (e.g., bent to a curvature radius) can be considered; see, e.g., Refs. 48,49.

Since recent studies on explanted stents obtained from human autopsy retrievals have also revealed in-vivo corrosion of stents, with significant release of metallic ions into surrounding tissues (see Refs. 48,49 and references therein), tests including mechanisms as pitting, fretting, crevice, and galvanic corrosion are recommended. Corrosion studies on stents have been presented in experimental campaigns as in Refs. 48,49, while no computational corrosion models are available from the literature. For instance, it was shown in Refs. 48,49 that biomechanical factors such as arterial curvature combined with stent overlapping or different overlapping materials increase the incidence of wear and fatigue fracture, due to micromotions between the overlapping portions.

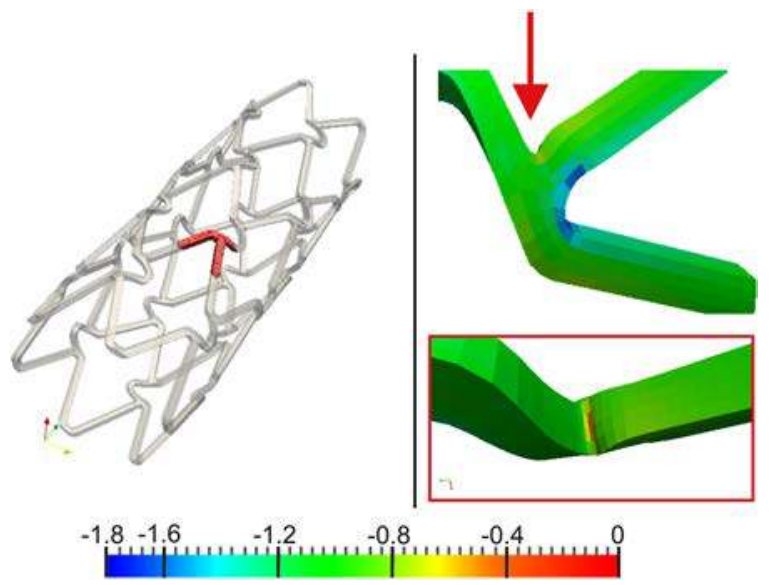

FIGURE 7. Fatigue-life assessment of a $316 \mathrm{~L}$ stainless steel Medinol NIR ${ }^{\mathrm{TM}}$ stent. Application of the Dang Van criterion to the stent strut (at the top). Isovalues of the Dang Van parameter (negative values indicate no failure; positive values indicate failure). Zoom of the region (at the bottom) where the critical points are located. Reprinted from Ref. 5, with permission from Elsevier.

Instead of accelerated durability testing, stress- or strain-based testing can be also performed on stents or representative specimens manufactured as stents, to provide fatigue performance information, constant life diagrams, and data for fatigue criteria calibration. ${ }^{47}$ Figure 6 shows several geometries of the representative specimens usually tested in the literature. ${ }^{66,74,82,88}$ The choice between stress- or strain-based testing requires an understanding of the in-vivo cyclic conditions and material type. ${ }^{19}$ Most Nitinol testing are strainbased, ${ }^{39,66,67,74}$ while most $316 \mathrm{~L}$ stainless steel and cobalt chromium testing are stress-based. ${ }^{23,55,82,88}$ Some limitations of these approaches have also to be considered: (i) due to size effects, specimens need to have dimensions comparable to those of stent components; ${ }^{81}$ (ii) not all the manufacturing process can be reproduced; (iii) complex in-vivo loading conditions (e.g., bending) are difficult to simulate. 


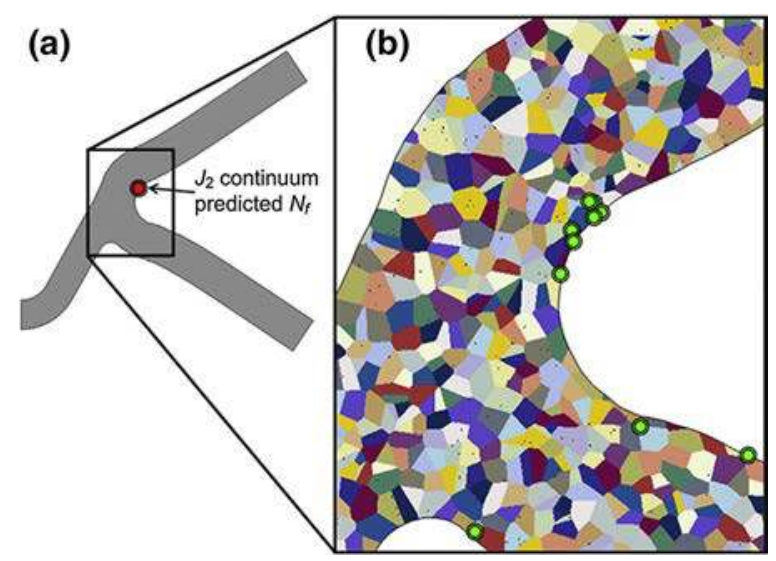

FIGURE 8. Fatigue-life assessment of a cobalt chromium Medinol NIR ${ }^{\mathrm{TM}}$ stent. (a) Predicted site of fatigue failure for the $J_{2}$ continuum sub-model and (b) predicted sites of fatigue crack initiation for the crystal plasticity sub-model. Reprinted from Ref. 82, with permission from Elsevier.

\section{NUMERICAL EVIDENCE}

This section discusses the results of the computational fatigue approaches available from the literature, by highlighting the main factors affecting stent lifetime.

Numerical results from the literature show that high stresses/strains usually develop near the most curved region of a stent strut due to stress concentration effects and small sizes, ${ }^{5,12,42,43,53,58,79,82}$ in accordance with experimental evidences. ${ }^{48,53}$ Such zones are the most vulnerable to the risk of long-term fatigue failure and they present the highest fatigue indicator factors (see Figs. 7 and 8).

The first factor affecting stent lifetime is the applied cyclic loading condition. Fatigue experiments, e.g., Ref. 66, have shown in fact that stresses and strains imposed by pulsatile loading are not the major responsible for SF. In accordance to these experimental observations, computational results have quantified bending loading 5 and cardiac wall movements ${ }^{58}$ as more dangerous than pulsatile loading; the diastole-systole loading have been shown to impose only very small changes of the strut diameter, which correspond to very small amplitudes for the cyclic stresses. ${ }^{5}$ Axial compression has been revealed more critical than bending for superficial femoral and popliteal artery stents. ${ }^{24}$

The second factor affecting stent lifetime is the plaque morphology, ${ }^{24}$ since the vessel thickness influences the local stiffness. Small thicknesses can easily shorten or bend, implying an increasing alternating strain and a decreasing mean strain. Figure 9 shows that the mean strain distribution caused by cyclic axial compression is similar for the considered plaques, due to the equal stenosis rate, while the alternating strain (a)

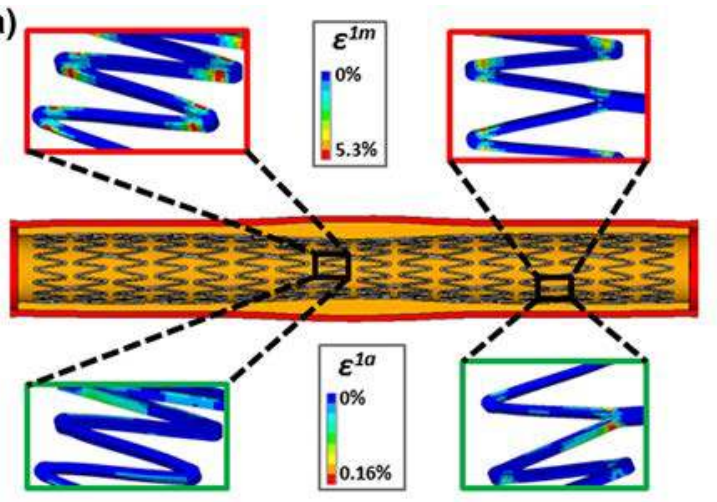

(b)

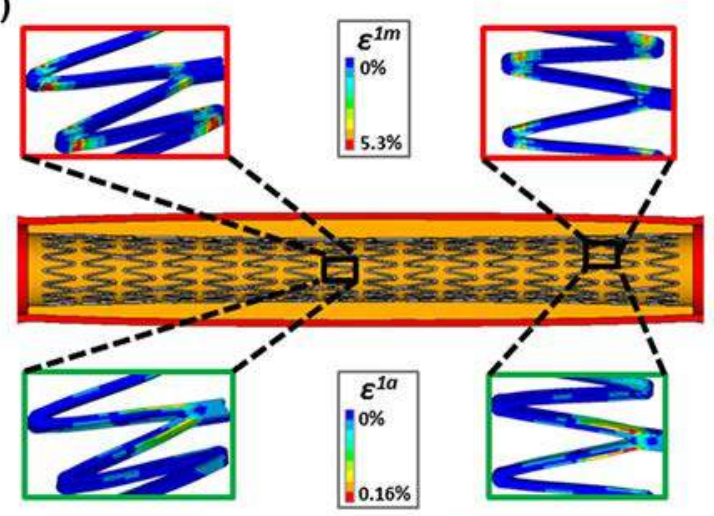

(c)



FIGURE 9. Fatigue-life assessment of a Nitinol peripheral stent. Contour plots of mean and alternating first principal strain for each plaque shape in case of cyclic axial compression: (a) plaque with high sharpness; (b) plaque with low sharpness, and (c) plaque with a double peak. Values of mean and alternating strain are reported in the red and green boxes, respectively. Reprinted from Ref. 24, with permission from Elsevier.

distribution is influenced by the thickness and stiffness of the artery-plaque system: the maximum alternating strains are located where the plaque exhibits a smaller thickness. ${ }^{24}$ Results in Ref. 58 also confirm the increased risk of coronary SF associated to localized plaque calcifications, particularly for the points in the curved region of the struts near the calcification. Recall, however, that current regulations ${ }^{84}$ do not require vessel and plaque inclusion in the analysis. 
The third factor affecting lifetime is the stent design and configuration (e.g, overlapping). The strut length and width have revealed the most substantial impact on fatigue resistance, followed by wall thickness and radius. ${ }^{12,53}$ The Goodman analysis performed in Ref. 12 shows that an increase in length or decrease in width and thickness results in an increase of stent lifetime, while an increase in radius results in a moderate increase of fatigue-life. Stent oversizing has revealed an important impact on stent lifetime from both numeri$\mathrm{cal}^{11}$ and experimental ${ }^{66}$ results. Large oversizing and compliance was in fact needed to reach strains causing fracture during experimental testing. ${ }^{66}$

Few numerical results have been validated on experimental fatigue data. Li et al..$^{53}$ performed an accelerated fatigue testing on a cobalt chromium stent. The stent did not fracture, as correctly predicted by the conducted Goodman analysis. Slip band induced by cyclic loading were found at the curvature location of rings, as predicted by numerical simulations showing high alternating stress distributed at curvature areas. Petrini et al. ${ }^{68}$ performed experimental fatigue tests on a NiTi stent in the free-expanded configuration under several combination of mean and alternating axial displacement until $10^{5}$ cycles. Numerical simulations predicted correctly the experimental failure and also the rupture location in three over four cases.

The reported successful matching between numerical predictions and clinical/experimental observations encourages the employment of computational analysis as a powerful tool for providing theoretical evidence, supporting the design process, and optimizing the stent configuration.

Finally, we recall that the discussed results do not consider the influence of patient-specific aspects on stent fatigue resistance. The inclusion of patientspecific image-based geometries allows a better characterization of the geometrical features of coronary arteries in terms of vessel curvature, dimensions, and plaque composition. ${ }^{58}$ Moreover, patient-specific modeling can be used as a validation tool through a direct comparison with stents extracted from patients. However, these data are difficult to procure and this approach may be less applicable to the initial stages of stent fatigue design, prior to approval for in-vivo operation. ${ }^{81}$

\section{CONCLUSIONS}

This paper has presented a review on the fatigue problem in metallic stents.

The employment of numerical simulations in the prediction of stent lifetime allows advanced modeling of the stenting procedures and it could provide an excellent tool for the optimization of novel stent designs or materials (e.g., magnesium-based bioresorbable stents ${ }^{32,36}$ ) and for the improvement of their fatigue performance. Three-dimensional numerical procedures should however take into account for stentartery interaction, material behavior, mechanical loading, and aggressive environment. Moreover, to validate computational approaches, an accurate comparison with the results of purposely-designed experimental tests should be conducted. As a future perspective, the fatigue performances of stent materials could be compared on a common stent platform for a wide range of performance metrics. The comparison could be made through simulated bench testing, based on finite element modelling, as already proposed for monotonic testing and assessment in Ref. 35.

It is worth underlining three main considerations: (i) given the steady increase of life expectancy ${ }^{90}$ and the persistent prevalence of cardiovascular disease in Western Countries, ${ }^{62,77}$ it is reasonable to expect that the long-term efficacy of endovascular implants and their fatigue resistance will be still an important research and industrial issue; (ii) several numerical works have focused on the fatigue-life assessment of stent designs which are no longer in use; (iii) clinical trials on stents are often targeting short- and mediumterm outcomes (from 6 months up to 1 year after stenting), neglecting possible fatigue failures. All these considerations highlight the need and importance of having an updated literature and experimental data regarding stent fatigue from the different point of views, i.e., researchers, manufacturers, clinicians, to improve current stent designs and realize novel solutions.

\section{ELECTRONIC SUPPLEMENTARY MATERIAL}

The online version of this article (doi: $10.1007 / \mathrm{s} 10439-015-1447-8)$ contains supplementary material, which is available to authorized users.

\section{ACKNOWLEDGMENTS}

This work is partially funded by: the Cariplo Foundation through the Project No. 2009.2822; ERC Starting Grant through the Project ISOBIO: Isogeometric Methods for Biomechanics (No. 259229); Ministero dell'Istruzione, dell'Università e della Ricerca through the Project No. 2010BFXRHS; the French National Research Agency (Project Fast3DANR-11-BS09-012-01). 


\section{REFERENCES}

${ }^{1}$ Adlakha, S., M. Sheikh, J. Wu, M. W. Burket, U. Pandya, W. Colyer, E. Eltahawy, and C. J. Cooper. Stent fracture in the coronary and peripheral arteries. J. Interv. Cardiol. 23(4):411-419, 2010.

${ }^{2}$ Aghel, A., E. J. Armstrong. Recent advances in self-expanding stents for use in the superficial femoral and popliteal arteries. Expert Rev. Cardiovasc. Ther. 12(7):833842, 2014.

${ }^{3}$ AL-Mangour, B., R. Mongrain, and S. Yue. Coronary stents fracture: an engineering approach (review). Mater. Sci. Appl. 4(10):606-621, 2013.

${ }^{4}$ Amiable, S., S. Chapuliot, A. Constantinescu, and A. Fissolo. A comparison of lifetime prediction methods for a thermal fatigue experiment. Int. J. Fatigue 28(7):692-706, 2006.

${ }^{5}$ Auricchio, F., A. Constantinescu, M. Conti, and G. Scalet. A computational approach for the lifetime prediction of cardiovascular balloon-expandable stents. Int. J. Fatigue 75:69-79, 2015.

${ }^{6}$ Auricchio, F., A. Constantinescu, C. Menna, and G. Scalet. A shakedown analysis of high cycle fatigue of shape memory alloys. 2015 (submitted).

${ }^{7}$ Auricchio, F., A. Constantinescu, and G. Scalet. Fatigue of $316 \mathrm{~L}$ stainless steel notched $\mu \mathrm{m}$-size components. Int. J. Fatigue, 68:231-247, 2014.

${ }^{8}$ Auricchio, F., L. Petrini. A three-dimensional model describing stress-temperature induced solid phase transformations: solution algorithm and boundary value problems. Int. J. Numer. Methods Eng. 6:807-836, 2004.

${ }^{9}$ Auricchio, F., R. L. Taylor, and J. Lubliner. Shape-memory alloys: macromodelling and numerical simulations of the superelastic behavior. Comput. Methods Appl. Mech. Eng. 146:281-312, 1997.

${ }^{10}$ Azaouzi, M., A. Makradi, and S. Belouettar. Fatigue life prediction of cardiovascular stent using finite element method. Comput. Methods Biomech. Biomed. Eng. 15(S1):93-95, 2012.

${ }^{11}$ Azaouzi, M., A. Makradi, and S. Belouettar. Deployment of a self-expanding stent inside an artery: a finite element analysis. Mater. Des. 41:410-420, 2012.

${ }^{12}$ Azaouzi, M., A. Makradi, J. Petit, S. Belouettar, and O. Polit. On the numerical investigation of cardiovascular balloon-expandable stent using finite element method. Comput. Mater. Sci. 79:326-335, 2013.

${ }^{13}$ Barrera, O., Makradi. A., M. Abbadi, M. Azaouzi, and S. Belouettar. On high-cycle fatigue of $316 \mathrm{~L}$ stents. Comput. Methods Biomech. Biomed. Eng. 2014, 17(3):239-250.

${ }^{14}$ Bertolino, G., A. Constantinescu, M. Ferjani, and P. Treiber. A multiscale approach of fatigue and shakedown for notched structures. Theor. Appl. Fract. Mech. 48(2):140-151, 2007.

${ }^{15}$ Bessias, N., G. Sfyroeras, and K. G. Moulakakis. Renal artery thrombosis caused by stent fracture in a single kidney patient. J. Endovasc. Ther. 12:516-520, 2005.

${ }^{16}$ Chang, C. K., C. P. Huded, B. W. Nolan, and R. J. Powell. Prevalence and clinical significance of stent fracture and deformation following carotid artery stenting. J. Vasc. Surg. 54(3):685-90, 2011.

${ }^{17}$ Charkaluk, E., A. Constantinescu, F. Szmytka, and S. Tabibian. Probability density functions: from porosities to fatigue lifetime. Int. J. Fatigue 63:127-136, 2014.

${ }^{18}$ Cheng, C., G. Choi, R. Herfkens, and C. Taylor. The effect of aging on deformations of the superficial femoral artery resulting from hip and knee flexion: Potential clinical implications. J. Vasc. Interv. Radiol. 21(2):195-202, 2010.

${ }^{19}$ Chen, Q., G. A. Thouas. Metallic implant biomaterials. Mater. Sci. Eng. R Rep. 87:1-57, 2015.

${ }^{20}$ Chung, W. S., C. S. Park, K. B. Seung, P. J. Kim, J. M. Lee, B. K. Koo, Y. S. Jang, J. Y. Yang, J. H. Yoon, D. I. Kim, Y. W. Yoon, J. S. Park, Y. H. Cho, and S. J. Park. The incidence and clinical impact of stent strut fractures developed after drugeluting stent implantation. Int. J. Cardiol. 125(3):325-331, 2008.

${ }^{21}$ Constantinescu, A., K. Van Dang, and M. H. Maitournam. A unified approach for high and low cycle fatigue based on shakedown concepts. Fatigue Fract. Eng. Mater. Struct. 26:561-568, 2003.

${ }^{22}$ Coppi, G., R. Moratto, J. Veronesi, E. Nicolosi, and R. Silingardi. Carotid artery stent fracture identification and clinical relevance. J. Vasc. Surg. 51(6):1397-405, 2010.

${ }^{23}$ Donnelly, E. Geometry effect in the fatigue behaviour of microscale $316 \mathrm{~L}$ stainless steel specimens. $\mathrm{PhD}$ thesis, National University of Ireland, Galway, 2012.

${ }^{24}$ Dordoni, E., A. Meoli, W. Wu, G. Dubini, F. Migliavacca, G. Pennati, and L. Petrini. Fatigue behaviour of nitinol peripheral stents: the role of plaque shape studied with computational structural analyses. Med. Eng. Phys. 36(7):842-849, 2014.

${ }^{25}$ Dordoni, E., L. Petrini, W. Wu, F. Migliavacca, G. Dubini, and G. Pennati. Computational modeling to predict fatigue behavior of NiTi stents: what do we need? J. Funct. Biomater. 6(2):299, 2015.

${ }^{26}$ dos Santos, H. A. F., F. Auricchio, and M. Conti. Fatigue life assessment of cardiovascular balloon-expandable stents: a two-scale plasticity-damage model approach. $J$. Mech. Behav. Biomed. 15:78-92, 2012.

${ }^{27}$ Foerst, J., T. Ball, and A. V. Kaplan. Postmortem in situ micro-CT evaluation of coronary stent fracture. Catheter Cardiovasc. Interv. 76(4):527-31, 2010.

${ }^{28}$ Gall, K., H. Sehitoglu. The role of texture in tensioncompression asymmetry in polycrystalline NiTi. Int. J. Plast. 15:69-92, 1999.

${ }^{29}$ Garcia, L., M. R. Jaff, C. Metzger, G. Sedillo, A. Pershad, F. Zidar, R. Patlola, R. G. Wilkins, A. Espinoza, A. Iskander, et al. Wire-interwoven nitinol stent outcome in the superficial femoral and proximal popliteal arteries twelve-month results of the superb trial. Circ. Cardiovasc. Interv. 8(5): $0000937,2015$.

${ }^{30}$ Garcia-Toca, M., H. E. Rodriguez, P. A. Naughton, A. Keeling, S. V. Phade, M. D. Morasch, M. R. Kibbe, and M. K. Eskandari. Are carotid stent fractures clinically significant? Cardiovasc. Intervent Radiol. 35:263-267, 2012.

${ }^{31}$ Garg, S., P. W. Serruys. Coronary stents: current status. $J$. Am. Coll. Cardiol. 56(10):S1-S42, 2010.

${ }^{32}$ Gastaldi, D., V. Sassi, L. Petrini, M. Vedani, S. Trasatti, and F. Migliavacca. Continuum damage model for bioresorbable magnesium alloy devices - application to coronary stents. J. Mech. Behav. Biomed. Mater. 4:352-365, 2011.

${ }^{33}$ Glenn, R., J. Lee. Accelerated pulsite fatigue testing of NiTi coronary stents. In A. Pelton, D. Hodgson, S. Russell, and T. Duerig, editors, Proceedings of the Second International Conference on Shape Memory and Superelastic Technologies (SMST 1997). SMST Society, Pacific Grove, California, CA, USA, 1997.

${ }^{34}$ Gong, X., A. Pelton, T. Duerig, N. Rebelo, and K. Perry. Finite element analysis and experimental evaluation of superelastic nitinol stent. In Proceedings of the International 
Conference on Shape Memory and Superelastic Technologies (SMST2003), pp. 453-462, 2003.

${ }^{35}$ Grogan, J. A., S. B. Leen, and P. E. McHugh. Comparing coronary stent material performance on a common geometric platform through simulated bench testing. J. Mech. Behav. Biomed. Mater. 12:129-138, 2012.

${ }^{36}$ Grogan, J. A., S. B. Leen, and P. E. McHugh. Computational micromechanics of bioabsorbable magnesium stents. J. Mech. Behav. Biomed. Mater. 34:93-105, 2014.

${ }^{37}$ Guerchais, R., F. Morel, N. Saintier, and C. Robert. Influence of the microstructure and voids on the high-cycle fatigue strength of 3161 stainless steel under multiaxial loading. Fatigue Fract. Eng. Mater. Struct. 38(9):10871104, 2015.

${ }^{38}$ Halwani, D. O., P. P. G. Anderson, B. C. Brott, A. S. Anayiotos, and J. E. Lemons. The role of vascular calcificationin inducing fatigue and fracture of coronary stents. $J$. Biomed. Mater. Res. B Appl. Biomater. 100(1):292-304, 2012.

${ }^{39}$ Harvey, S. M. Nitinol stent fatigue in a peripheral human artery subjected to pulsatile and articulation loading. $J$. Mater. Eng. Perform. 20:697-705, 2011.

${ }^{40}$ W. Higashiura, Y. Kubota, S. Sakaguchi, N. Kurumatani, M. Nakamae, K. Nishimine, and K. Kichikawa. Prevalence, factors, and clinical impact of self-expanding stent fractures following iliac artery stenting. J. Vasc. Surg., 49(3):645-652, 2009.

${ }^{41}$ Hsiao, H. M., A. Nikanorov, S. Prabhu, and M. K. Razavi. Respiration-induced kidney motion on cobalt-chromium stent fatigue resistance. J. Biomed. Mater. Res. B Appl. Biomater. 91B(2):508-516, 2009.

${ }^{42}$ Hsiao, H. M., S. Prabhu, A. Nikanorov, and M. Razavi. Renal artery stent bending fatigue analysis. J. Med. Devices 1(2):113-118, 2006.

${ }^{43}$ Hsiao, H. M., M. T. Yin. An intriguing design concept to enhance the pulsatile fatigue life of self-expanding stents. Biomed. Microdevices 16(1):133-41, 2014.

${ }^{44} \mathrm{http}: / /$ worldwide.bose.com/electroforce.

${ }^{45}$ International Standard ISO 25539-2. Cardiovascular implants - endovascular devices_-Part 2: Vascular stents, 2012.

${ }^{46}$ Jabbado, M., H. Maitournam. A high-cycle fatigue life model for variable amplitude multiaxial loading. Fatigue Fract. Eng. Mater. Struct. 31(1):67-75, 2008.

${ }^{47}$ James, B. A., R. A. Sire. Fatigue-life assessment and validation techniques for metallic vascular implants. Biomaterials 31(2):181-186, 2010.

${ }^{48}$ Kapnisis, K., G. Constantinides, H. Georgiou, D. Cristea, C. Gabor, D. Munteanu, B. Brott, P. Anderson, J. Lemons, and A. Anayiotos. Multi-scale mechanical investigation of stainless steel and cobalt-chromium stents. J. Mech. Behav. Biomed. Mater. 40:240-251, 2014.

${ }^{49}$ Kapnisis, K. K., D. O. Halwani, B. C. Brott, P. G. Anderson, J. E. Lemons, and A. S. Anayiotos. Stent overlapping and geometric curvature influence the structural integrity and surface characteristics of coronary nitinol stents. J. Mech. Behav. Biomed. Mater. 20:227-236, 2013.

${ }^{50}$ Kinkel, S., N. Wollmerstedt, J. A. Kleinhans, C. Hendrich, and C. Heisel. Patient activity after total hip arthroplasty declines with advancing age. Clin. Orthop. Relat. Res. 467(8):2053-2058, 2009.

${ }^{51}$ Lagoudas, D. C., D. J. Hartl, Y. Chemisky, L. Machado, and P. Popov. Constitutive model for the numerical anal- ysis of phase transformation in polycrystalline shape memory alloys. Int. J. Plast. 32-33:155-183, 2012.

${ }^{52}$ Lewitton, S., A. Babaev. Superficial femoral artery stent fracture that led to perforation, hematoma and deep venous thrombosis. J. Invasive Cardiol. 20(9):479-81, 2008.

${ }^{53} \mathrm{Li}$, J., Q. Luo, Z. Xie, Y. Li, and Y. Zeng. Fatigue life analysis and experimental verification of coronary stent. Heart Vessels 25(4):333-337, 2010.

${ }^{54}$ Lin, Y., X. Tang, W. Fu, R. Kovach, J. C. George, and D. Guo. Stent fractures after superficial femoral artery stenting: risk factors and impact on patency. J. Endovasc. Ther. 22(3):319-326, 2015.

${ }^{55}$ Marrey, R. V., R. Burgermeister, R. B. Grishaber, and R. O. Ritchie. Fatigue and life prediction for cobalt-chromium stents: a fracture mechanics analysis. Biomaterials 27:19882000, 2006.

${ }^{56}$ McGarry, J. P., B. P. O’Donnell, P. E. McHugh, and J. G. McGarry. Analysis of the mechanical performance of a cardiovascular stent design based on micromechanical modelling. Comput. Mater. Sci. 31:421-438, 2004.

${ }^{57}$ Meoli, A., E. Dordoni, L. Petrini, F. Migliavacca, G. Dubini, and G. Pennati. Computational study of axial fatigue for peripheral nitinol stents. J. Mater. Eng. Perform. 23(7):2606-2613, 2014.

${ }^{58}$ Morlacchi, S., G. Pennati, L. Petrini, G. Dubini, and F. Migliavacca. Influence of plaque calcifications on coronary stent fracture: a numerical fatigue life analysis including cardiac wall movement. J. Biomech. 47(4):899-907, 2014.

${ }^{59}$ Z. Moumni, W. Zaki, and H. Maitournam. Cyclic behaviour and energy approach of the fatigue of Shape Memory Alloys. J. Mech. Mater. Struct., 4(2):395-411, 2009.

${ }^{60}$ Müller-Hülsbeck, S., P. J. Schäfer, N. Charalambous, H. Yagi, M. Heller, and T. Jahnke. Comparison of secondgeneration stents for application in the superficial femoral artery: an in vitro evaluation focusing on stent design. $J$. Endovasc. Ther. 17(6):767-776, 2010.

${ }^{61}$ Neil, N. Stent fracture in the superficial femoral and proximal popliteal arteries: literature summary and economic impacts. Perspect. Vasc. Surg. Endovasc. Ther. 25(12):20-27, 2013

${ }^{62}$ Nichols, M., N. Townsend, P. Scarborough, and M. Rayner. Cardiovascular disease in europe 2014: epidemiological update. Eur. Heart J. 35(42):2950-9, 2014.

${ }^{63}$ Nikanorov, A., H. B. Smouse, K. Osman, M. Bialas, S. Shrivastava, and L. B. Schwartz. Fracture of self-expanding nitinol stents stressed in vitro under simulated intravascular conditions. J. Vasc. Surg. 48(2):435-440, 2008.

${ }^{64}$ Paulsen, F., J. Waschke. Sobotta Atlas of Human Anatomy, 15th Edition $2013{ }^{\circledR}$ Elsevier GmbH, Urban \& Fischer, Munich.

${ }^{65}$ Peigney, M. Shakedown theorems and asymptotic behaviour of solids in non-smooth mechanics. Eur. J. Mech. A 29(5):784-793, 2010.

${ }^{66}$ Pelton, A. R., V. Schroeder, M. R. Mitchell, X.-Y. Gong, M. Barney, and S. W. Robertson. Fatigue and durability of Nitinol stents. J. Mech. Behav. Biomed. 1:153-164, 2008.

${ }^{67}$ Petrini, L., W. Wu, E. Dordoni, A. Meoli, F. Migliavacca, and G. Pennati. Fatigue behavior characterization of nitinol for peripheral stents. Funct. Mater. Lett. 05(01):1250012, 2012.

${ }^{68}$ Petrini, L., E. Dordoni, W. Wu, C. Guala, C. Silvestro, F. Migliavacca, and G. Pennati. Fatigue resistance of Nitinol peripheral stents. In Proceedings of 6th ECCOMAS 
Conference on Smart Structures and Materials (SMART 2013), 2013

${ }^{69}$ Rebelo, N., A. Zipse, M. Schlun, and G. Dreher. A material model for the cyclic behavior of nitinol. J. Mater. Eng. Perform. 20:605-612, 2011

${ }^{70}$ Robertson, S. W., C. P. Cheng, and M. K. Razavi. Biomechanical response of stented carotid arteries to swallowing and neck motion. J. Endovasc Ther. 15(6):66371, 2008.

${ }^{71}$ Robertson, S. W., D. B. Jessup, I. J. Boero, and C. P. Cheng. Right renal artery in vivo stent fracture. J. Vasc. Interv. Radiol. 19(3):439-442, 2008.

${ }^{72}$ Robertson, S. W., A. R. Pelton, and R. O. Ritchie. Mechanical fatigue and fracture of Nitinol. Int. Mater. Rev. 57(1):1-37, 2012.

${ }^{73}$ Robertson, S. W., R. O. Ritchie. A fracture-mechanicsbased approach to fracture control in biomedical devices manufactured from superelastic Nitinol tube. J. Biomed. Mater. Res. B 84B(1):26-33, 2008.

${ }^{74}$ Runciman, A., D. Xu, A. R. Pelton, and R. O. Ritchie. An equivalent strain/Coffin-Manson approach to multiaxial fatigue and life prediction in superelastic Nitinol medical devices. Biomaterials 32:4987-4993, 2011.

${ }^{75}$ Scheinert, D., S. Scheinert, J. Sax, C. Piorkowski, S. Braunlich, and M. Ulrich. Prevalence and clinical impact of stent fractures after femoropopliteal stenting. J. Am. Coll. Cardiol. 45:312-315, 2005.

${ }^{77}$ Shechter, G., J. R. Resar, and E. R. McVeigh. Displacement and velocity of the coronary arteries: cardiac and respiratory motion. IEEE Trans. Med. Imaging 25(3):369375, 2006.

${ }^{76}$ Sources: Cdc.gov-heart disease facts american heart association-2015 heart disease and stroke update, compiled by aha, cdc, nih and other governmental sources, 2014.

${ }^{78}$ Souza, A. C., E. N. Mamiya, and N. Zouain. Three-dimensional model for solids undergoing stress-induced phase transformations. Eur. J. Mech. A-Solid 17:789-806, 1998.

${ }^{79}$ Sweeney, C. A., P. E. McHugh, J. P. McGarry, and S. B. Leen. Micromechanical methodology for fatigue in cardiovascular stents. Int. J. Fatigue 44:202-216, 2012.
${ }^{80}$ Sweeney, C. A., B. O’Brien, F. P. E. Dunne, P. E. McHugh, and S. B. Leen. Strain-gradient modelling of grain size effects on fatigue of cocr alloy. Acta Mater. 78:341-353, 2014.

${ }^{81}$ Sweeney, C. A., B. O’Brien, F. P. E. Dunne, P. E. McHugh, and S. B. Leen. Micro-scale testing and micromechanical modelling for high cycle fatigue of cocr stent material. $J$. Mech. Behav. Biomed. Mater. 46:244-260, 2015.

${ }^{82}$ Sweeney, C. A., B. O'Brien, P. E. McHugh, and S. B. Leen. Experimental characterisation for micromechanical modelling of CoCr stent fatigue. Biomaterials 35:36-48, 2014.

${ }^{83}$ Tabanli, R. M., N. K. Simha, and B. T. Berg. Mean strain effects on the fatigue properties of superelastic NiTi. Metall. Mater. Trans. A 32:1866-1869, 2001.

${ }^{84}$ US Food and Drug Administration. Non-clinical engineering tests and recommended labeling for intravascular stents and associated delivery systems: guidance for industry and FDA staff. US Department of Health and Human Services; Food and Drug Administration, Centre for Devices and Radiological, Health, April 18th 2010.

${ }^{85}$ Van Dang, K. High-cycle metal fatigue in the context of mechanical design. In: CISM Courses and Lectures no 392 edited by K. Dang Van and I. V. Papadopoulos. Springer, 57-88, 1999.

${ }^{86}$ Weafer, F. M., M. S. Bruzzi. Influence of microstructure on the performance of nitinol: a computational analysis. $J$. Mater. Eng. Perform. 23(7):2539-2544, 2014.

${ }^{87}$ Weiss, S., H. Szymczak, and A. Meissner. Fatigue and endurance of coronary stents. Materialwissenschaft und Werkstofftechnik 40(1-2):61-64, 2009.

${ }^{88}$ Wiersma, S., F. Dolan, and D. Taylor. Fatigue and fracture in materials used for micro-scale biomedical components. Biomed. Mater. Eng. 16(2):137-146, 2006.

${ }^{89}$ Wiersma, S., D. Taylor. Fatigue of materials used in microscopic components. Fatigue Fract. Eng. Mater. Struct. 28(12):1153-1160, 2005.

${ }^{90}$ World Health Organization (WHO). World Health Statistics 2014. Technical Report, 2014.

${ }^{91}$ Zaki, W., Z. Moumni. A 3D model of the cyclic thermomechanical behavior of shape memory alloys. J. Mech. Phys. Solids 55(11):2427-2454, 2007. 